\title{
Ueber Extrauterinschwangerschaft.
}

Von

\author{
Dr. Y. Iwase,
}

aus Tokyo, Japan.

Schon seit mehreren Jahrzehnten haben sich die Gynäkologen Europas eingehend mit dem umfangreichen und interessanten Kapitel der Extrauteringravidität beschäftigt, und hat sich besonders in Deutschland die Literatur hierüber ausserordentlich angehäuft. Unsere Kenntnisse über Aetiologie, Symptomatologie und Therapie der Erkrankung sind daher ziemlich reiche. Indessen dürfte es von Interesse sein zu untersuchen, ob bei anderen Völkerrassen die Verhältnisse dieselben sind wie in Europa, ob die Rassenunterschiede auch Unterschiede im Auftreten und der Form des Krankheitsbildes bedingen. Ich verfüge über 38 Fälle von Tubargravidität, die ich während der Jahre 1901-1906 in der Kaiserlich japanischen Universitäts-Frauenklinik zu Tokyo bei Prof. Dr. S. Kinoshita gesehen habe. Diese Zahl ist zwar zu klein, um daraus sichere Schlüsse ziehen zu können, doch möge sie als eine vorläufige Mittheilung genügen, die ich später ergänzen möchte.

Meine Fälle vertheilen sich auf die einzelnen Jahre folgendermaassen: 2 Fälle im Jahre 1901, 1 im Jahre 1902, 7 im Jahre 1903, 12 im Jahre 1904, 10 im Jahre 1905 und 6 Fälle im Jahre 1906.

\section{Alter der Patientin.}

Was das Alter der Patientinnen betrifft, so stand die jüngste im 24. Lebensjahr, die älteste im 39., wie auch aus den Tabellen. 1 und 2 ersichtlich ist. Am häufigsten trat die Extrauteringravidität im Alter von 25 bis 30 Jahren auf (14 Fälle unter 38, gleich 36,8 pCt.). Aehnliche Verhältnisse finde ich unter den deutschen Statistiken, bei Runge (1), der für diese Lebensperiode $33 \mathrm{pCt}$. 
berechnet, Dobbert(2) mit 23,3 pCt. Dann folgt in meinen Fällen das Alter zwischen 30 und 35 Jahren mit 11 Fällen, 28,9 pCt., welchesjedoch bei Martin (3), Küstner (4) und Seboll gerade das Prädilectionsalter für die ektopische Schwangerschaft darstellt. Bei der

\section{Alter.}

\begin{tabular}{|c|c|c|c|c|c|c|c|c|}
\hline \multirow{2}{*}{\multicolumn{2}{|c|}{ Alter }} & \multicolumn{7}{|c|}{ A utorname } \\
\hline & & Iwase & Dührssen & Martin & Küstner & Runge & Dobbert & Scholl \\
\hline 20 & Jahre & - & - & 1 & 2 & 5 & & \\
\hline 21 & $"$ & - & - & 1 & - & 4 & & \\
\hline 22 & $"$ & - & - & 1 & - & 9 & 8 & 14 \\
\hline 23 & $n$ & - & - & 2 & 2 & 13 & & \\
\hline $\begin{array}{l}24 \\
25\end{array}$ & $"$ & $\begin{array}{l}2 \\
4\end{array}$ & 1 & 2 & 5 & $\begin{array}{l}9 \\
9\end{array}$ & & \\
\hline & & & & & & & & \\
\hline 26 & $"$ & 2 & 1 & 1 & 3 & 13 & & \\
\hline 27 & $"$ & 4 & 2 & 6 & 4 & 16 & & \\
\hline 28 & 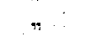 & 2 & 1 & 9 & 5 & 16 & 23 & 26 \\
\hline 29 & 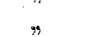 & 1 & 4 & 4 & 7 & 14 & & \\
\hline 30 & " & 5 & 2 & 2 & 9 & 17 & & \\
\hline 31 & 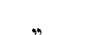 & 4 & 2 & 1 & 6 & 9 & & \\
\hline 32 & $"$ & 3 & 4 & 6 & 10 & 14 & & \\
\hline 33 & $"$ & 2 & 3 & 6 & 8 & 11 & 19 & 37 \\
\hline 34 & $"$ & 1 & 1 & 6 & 4 & 10 & & \\
\hline 35 & $"$ & 1 & 4 & 4 & 9 & 4 & & \\
\hline 36 & 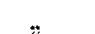 & 3 & 1 & 3 & 6 & 18 & & \\
\hline 37 & $"$ & 1 & 4 & 5 & 6 & 10 & & \\
\hline 38 & $"$ & - & - & 3 & 4 & 5 & 9 & 24 \\
\hline 39 & $"$ & 3 & 1 & 3 & 5 & 5 & & \\
\hline 40 & $"$ & - & - & - & 2 & 5 & & \\
\hline 41 & 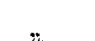 & - & - & 1 & 3 & 1 & & \\
\hline 42 & " & - & - & 1 & - & 4 & & \\
\hline 43 & , & 一 & - & - & 1 & 6 & 1 & 7 \\
\hline 44 & " & - & 1 & 1 & 1 & 1 & & \\
\hline 45 & $"$ & - & - & 1 & - & - & & \\
\hline 46 & 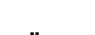 & _. & $\ldots$ & _- & - & 1 & & \\
\hline 48 & $"$ & - & - & - & - & 1 & & \\
\hline 53 &. & 一 & 一 & 1 & - & - & & \\
\hline$\tilde{5} 4$ & 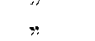 & - & - & - & 1 & - & & \\
\hline 55 & $"$ & - & - & 1 & - & - & & \\
\hline & & & & & & & & \\
\hline ohn & itstalter & 6 Mon. & 32 Jahre & $\begin{array}{l}32 \text { J. } \\
6 \text { Non. }\end{array}$ & $\begin{array}{l}32 \text { Jahre } \\
10 \text { Monate }\end{array}$ & 9 Mon. & & \\
\hline
\end{tabular}

geringen Zahl meiner Fälle fehlen die Jahre unter 23 und über 40; doch ist wohl anzunehmen, dass auch in Japan ausserhalb dieser Altersgrenzen Fälle von Extrauterin-Schwangerschaft vorkommen. Das durchschnittliche Alter meiner Patientinnen betrug 30 Jahre 
6 Monate, etwas weniger als bei den deutschen Autoren, wie ja auch das Praedilectionsalter mehr nach vorn gerückt war. So finden Martin (3) 32 Jahre 6 Monate, Küstner (4) 32 Jahre 10 Monate, Runge (1) 30 Jahre 9 Monate und Dührssen (5) 32 Jahre 4 Monate.

\section{Jahre.}

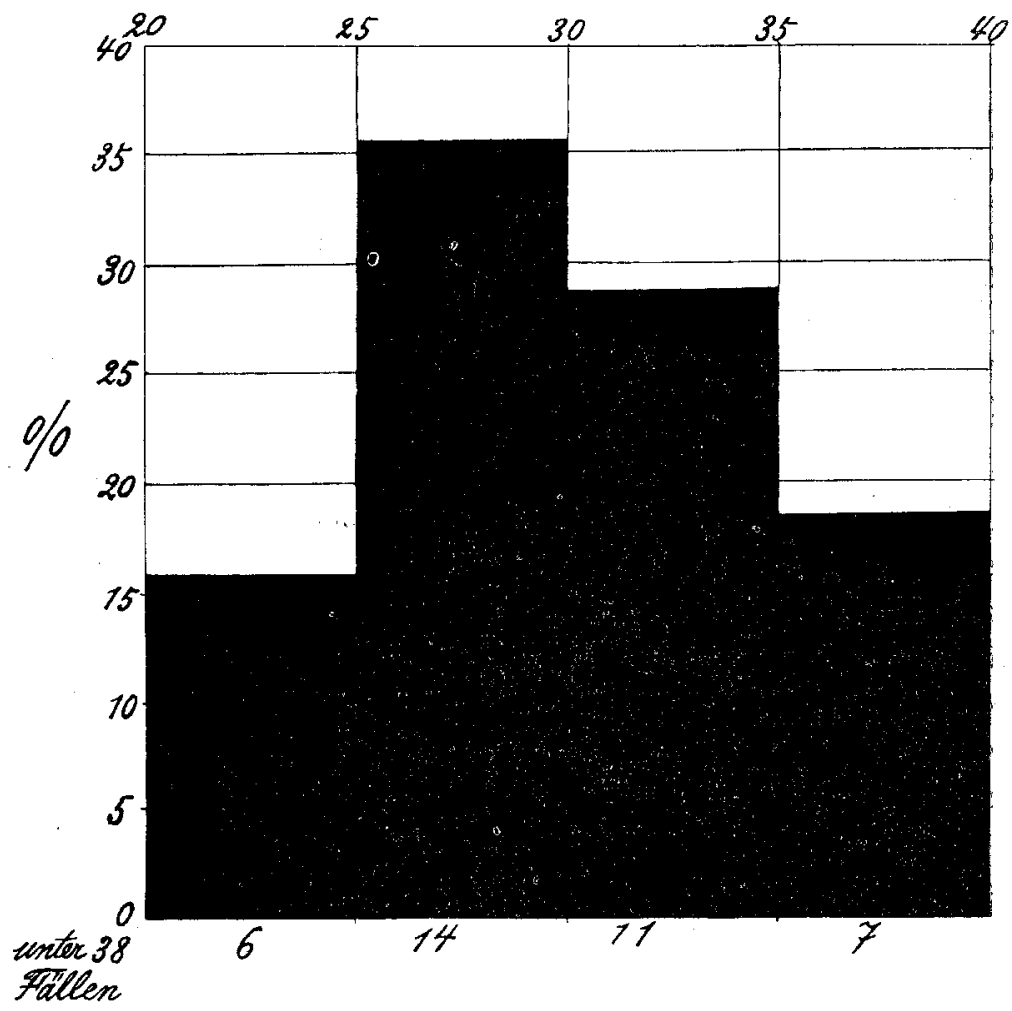

\section{Zahl der Geburten.}

Von verschiedenen Seiten wurde hervorgehoben, dass Erauen, die geboren haben, häufiger von Extrauteringravidität befallen werden, als Nullipara. Unter meinen 38 Fällen sind 33 Mehrgebärende $=86,8$ pCt. (s. Tabelle 3); Runge (1) berechnete 85,5 pCt., Moebius und Sittner (6) 87,1 pCt., Küstner (4) 89,2 pCt., Martin (3) 89,9 und Dobbert (2) 93,1 pCt. Die Durchschnittszahl der vorausgegangenen Geburten beträgt in meinen Fällen 1,86.

Besonders auffällig ist dabei die Häufigkeit der Erkrankung bei Frauen, die nur einmal geboren haben, nämlich 15 unter 38 
III. Zah1 der Geburten.

\begin{tabular}{|c|c|c|c|c|c|c|c|}
\hline \multirow{2}{*}{ Para } & \multicolumn{7}{|c|}{ A u torna $\mathrm{me}$} \\
\hline & Iwase & Martin & Dobbert & $\begin{array}{l}\text { Moebius } \\
\text { u. Sittner }\end{array}$ & Küstner & Runge & Dührssen \\
\hline 0 & 5 & 7 & 4 & 15 & 11 & 33 & 8 \\
\hline $1 \mathrm{mal}$ & 15 & 17 & 15 & 37 & 26 & 61 & 9 \\
\hline 2 & 5 & 16 & 18 & 29 & 22 & 42 & 10 \\
\hline 3 & 8 & 10 & 12 & 11 & 10 & 23 & 1 \\
\hline $4 n$ & 4 & 6 & 5 & 10 & 11 & 18 & 3 \\
\hline 5 & - & 3 & - & 6 & 6 & 22 & 1 \\
\hline 6 & 1 & 4 & 2 & 5 & 3 & 8 & - \\
\hline 7 & - & 3 & 1 & 2 & 4 & 7 & 1 \\
\hline $8 \%$ & - & 1 & 1 & 1 & 5 & 1 & 2 \\
\hline 9 & - & - & - & - & - & 4 & -- \\
\hline $10 "$ & - & 2 & - & 一 & 2 & $\dot{0}$ & - \\
\hline $11 \%$ & - & 一 & $\cdots$ & - & 2 & 1 & 一 \\
\hline 12 & 一 & - & - & 1 & 1 & 2 & - \\
\hline $14 "$ & - & - & - & - & - & 1 & - \\
\hline $\begin{array}{l}\text { Duren- } \\
\text { schnitts- } \\
\text { geburtszahi }\end{array}$ & 1,86 & 2,7 & 2,3 & 2,2 & 3 & 2,8 & 2 \\
\hline
\end{tabular}

(=39,5 pCt). Während von diesen 12 Frauen schon innerhalb 3 Jahren nach der Verheirathung geboren hatten, dauerte bei ihnen die Zeit von da bis zum Eintritt der ektopischen Gravidität sehr lange, im Mittel 7 Jahre. Daraus und aus der oben erwähnten Häufigkeit der Erkrankung bei Mehrgebärenden lässt sich, wie auch von anderer Seite betont wurde, der Schluss ziehen, dass die Geburt oder das Wochenbett einen wichtigen Factor für die Entstehung des Leidens bildet.

Ebenso wichtig ist aber auch eine vorhergegangene Frühgeburt oder ein Abort, bei denen durch mangelhafte Rückbildung oder puerperale Infection Genitalleiden entstehen, die eine ektopische Ansiedelung des Eies im Gefolge haben können. So habe ich unter meinem Material 10 Fälle (= 26,3 pCt.) von Abort oder Frühgeburt; Küstner (4) hatte 26,1 pCt., Dührssen (5) 16,7 pCt. Besonders bemerkenswert ist, dass von diesen 10 Fällen 9 mal die Fehlgeburt der Extrauteringravidität unmittelbar voranging, wie auch bei Dührssen unter 5 Fällen 4 mal die Fehlgeburt als letzte eingetreten war. Daraus lässt sich wohl folgen, dass die Frühgeburt oder der Abort bei der Entstehung der Tubenschwangerschaft eine Rolle spielt, dadurch, dass sie ein organisches Genitalleiden verursacht. Andererseits kann man auch annehmen, dass bereits die vorangegangene Fehlgeburt durch dasselbe Genitalleiden bedingt wurde, welches nachher die Extrauterinschwangerschaft auslöste. So in meinem Fall 21, in welchem unter 6 Schwanger- 
schaften nur die erste das normale Ende erreichte, während die übrigen $5 \mathrm{im}$ 3.-4. Monat durch uterinen Abort endeten, worauf dann die Tubengravidität folgte.

\section{Die nach der letzten Geburt verflossene Zeit.}

Wie Tabelle 4 zeigt, betrug die kürzeste Zeit nach der zuletzt beendeten Gravidität bis zum Eintritt der ektopischen SchwangerIV. Die nach der letzten Geburt verflossene Zeit.

\begin{tabular}{|c|c|c|c|c|c|c|c|}
\hline Zeit & Iwase & Dührssen & Martin & $\begin{array}{l}\text { torn } \\
\text { Dobbert }\end{array}$ & $\begin{array}{l}\text { a } m e \\
\text { Moebius } \\
\text { a. Sittner }\end{array}$ & Küstner & Runge \\
\hline 3 Monate & - & - & - & - & 一 & 一 & 7 \\
\hline $4 \quad "$ & - & $i$ & - & - & - & 一 & - \\
\hline $5 \quad "$ & - & 1 & 1 & - & - & - & 10 \\
\hline $6 \quad:$ & - & 2 & 1 & 2 & - & - & - \\
\hline 7 & 1 & 1 & 1 & - & - & 3 & - \\
\hline 8 & 一 & 1 & - & - & - & 3 & 9 \\
\hline 9 & 一 & - & 1 & - & 1 & 3 & - \\
\hline 10 & 1 & - & - & 一 & 1 & - & - \\
\hline $11 \%$ & - & - & 1 & - & 1 & 2 & 20 \\
\hline 1 Jahr & 3 & 2 & 4 & 4 & 1 & 2 & 10 \\
\hline $1 \mathrm{~J} .3 \mathrm{M}$. & 一 & - & 2 & - & - & 4 & $\ldots$ \\
\hline $1,4 \%$ & 2 & - & - & - & - & - & 8 \\
\hline $1 \% 6 \%$ & - & 2 & 2 & 3 & 1 & 4 & 一 \\
\hline $1,8 n$ & - & - & - & - & 1 & 一 & 1 \\
\hline $1,9 n$ & - & - & 1 & - & 1 & 1 & 12 \\
\hline 2 Jahre & 2 & 1 & 2 & 5 & 10 & 8 & - \\
\hline 2 J. 3 M. & - & - & - & - & - & 1 & 5 \\
\hline $2,6 \%$ & 1 & - & 1 & - & 3 & 5 & - \\
\hline $2,9 \geqslant$ & 一 & - & - & - & - & 1 & 19 \\
\hline 3 Jahre & 2 & 3 & 1 & 8 & 3 & 7 & - \\
\hline $3 \mathrm{J.} 6 \mathrm{M.}$ & 一 & 3 & 1 & 一 & 1 & - & 8 \\
\hline 4 Jahre & 1 & 4 & 3 & 4 & 4 & 8 & - \\
\hline 4 J. 6 M. & - & - & - & - & 一 & 1 & 9 \\
\hline 5 Jahre & 2 & 1 & 5 & 6 & 9 & 3 & 12 \\
\hline $6 \%$ & 4 & - & 2. & 3 & 6 & 7 & - \\
\hline $6 \mathrm{~J} .6 \mathrm{M}$ & 一 & - & 1 & - & - & - & 8 \\
\hline 7 Jahre & 4 & 2 & 2 & 2 & 4 & 3 & 12 \\
\hline 8 & 1 & 1 & 1 & -- & 6 & 一 & 14 \\
\hline 9 & - & - & 2 & 3 & 5 & 2 & 3 \\
\hline 10 & 3 & 一 & 1 & 1 & 6 & 3 & 1 \\
\hline 11 & 1 & - & 1 & 2 & 2 & 2 & 1 \\
\hline 12 & 3 & - & 4 & -1 & - & 4 & 3 \\
\hline 13 & 1 & - & - & 1 & 5 & 3 & 1 \\
\hline 14 & - & - & - & 1 & 3 & 1 & - \\
\hline 15 & - & 1 & 2 & 2 & 2 & - & 1 \\
\hline 16 & 1 & - & - & 1 & - & - & 2 \\
\hline 17 & - & - & - & 1 & 1 & - & - \\
\hline 19 & - & - & - & - & 1 & - & - \\
\hline 20 & - & - & 1 & - & 1 & - & - \\
\hline 22 & - & 一 & - & - & - & 1 & - \\
\hline $\begin{array}{l}\text { Durch- } \\
\text { schnitt }\end{array}$ & 6 Jabre & $\begin{array}{l}\text { 4 Jahre } \\
3 \text { Monate }\end{array}$ & $\begin{array}{l}5 \text { Jahre } \\
6 \text { Mon. }\end{array}$ & $\begin{array}{l}5 \text { Jahre } \\
6 \text { Monate }\end{array}$ & $\begin{aligned} 6 & \text { Jahre } \\
10 & \text { Monate }\end{aligned}$ & $\begin{array}{l}4 \text { Jahre } \\
8 \text { Monate }\end{array}$ & 4 Jahre \\
\hline
\end{tabular}


schaft 7 Monate, die längste 16 Jahre; im Mittel 6 Jahre. Aehnlich sind die Angaben der deutschen Autoren: Runge (1) berechnete 4 Jahre. Küstner (4) 4 Jahre 8 Monate, Wormser (9) 4 Jahre 5 Monate, Dührssen (5) 4 Jahre 3 Monate, Martin (3) sowie Dobbert (2) 5 Jahre 6 Monate, Moebius und Sittner (6) endlich 6 Jahre 10 Monate. Ich habe diese Zahlen in Tabelle 4 zusammengestellt.

Bei den meisten Frauen liegt die Zeit der letzten Geburt über 2 Jahre zurück, in meinen Fällen 26 unter $33=78,8$ pCt. Auch Runge (1) hat unter 176 Mehrgebärenden $111=63 \mathrm{pCt}$, bei denen bereits über 2 Jahre nach der letzten Geburt verflossen sind; Martin (3) 30 unter $44=68,2$ pCt., Küstner (4) 60 unter $82=73,1$ pCt., Moebius und Sittner (6) 62 unter 79 gleich 83,5 pCt., Dobbert (2) 44 unter $49=81,6$ pCt.

Sehr interessant ist, dass auch bei Nulliparen die Zeit bis zum Eintritt der Tubenschwangerschaft eine sehr lange ist; die Frauen waren in meinen Fällen 2, 5, 8, 12 und 16 Jahre steril verheirathet; im Durchschnitt 8 Jahre 7 Monate. Es ist also auch hier, wie bei den Mehrgebärenden, eine relative Sterilität vorhanden, deren Ursachen wohl sicher zugleich als ätiologische Momente für die Tubengravidität in Betracht kommen.

\section{Seite der Extranteringravidität.}

Hinsichtlich der Seite, in der sich die ektopische Schwangerschaft entwickelt, zeigt sich keine besondere Vorliebe für rechts oder links. Es wird in gleicher Weise die rechte und die linke

V. Seite der Extrauteringravidität.

\begin{tabular}{|c|c|c|c|}
\hline Autorname & rechts & links & $\begin{array}{c}\text { Proportion } \\
\text { rechts } \\
\text { zu links }\end{array}$ \\
\hline 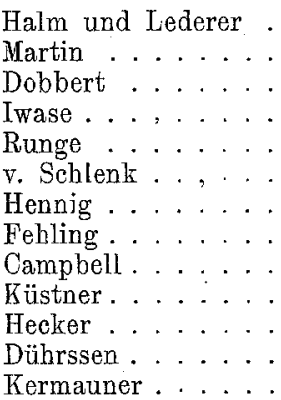 & $\begin{array}{r}17 \\
44 \\
32 \\
20 \\
106 \\
124 \\
61 \\
19 \\
34 \\
46 \\
27 \\
16 \\
14\end{array}$ & $\begin{array}{r}9 \\
33 \\
24 \\
18 \\
94 \\
123 \\
61 \\
24 \\
41 \\
52 \\
37 \\
22 \\
25\end{array}$ & $\begin{aligned} 1,9 & : 1 \\
1,33 & : 1 \\
1,33 & : 1 \\
1,11 & : 1 \\
1,11 & : 1 \\
1,008 & : 1 \\
1 & : 1 \\
1 & : 1,2 \\
1 & : 1,2 \\
1 & : 1,13 \\
1 & : 1,37 \\
1 & : 1,4 \\
1 & : 1,78\end{aligned}$ \\
\hline
\end{tabular}


Tube betroffen, wie auch Werth (10) in Winckel's Handbuch hervorgehoben hat und auch aus Tabelle 5 hervorgeht. In meinen Fällen war $20 \mathrm{mal}$ die rechte, $18 \mathrm{mal}$ die linke Tube schwanger, also im Verhältnis von $1,11: 1,0$.

\section{In ätiologischer Beziehung}

sind mir besonders 2 Fälle von Wichtigkeit (Fall 8 und 17), bei denen die ektopische Schwangerschaft kurz nach einer RetroversioOperation eintrat. Ich finde bisher nur einen solchen Fall von Küstner (4) beschrieben. Er schreibt: „Ein Fall von Retroflexion, in welchem Sterilität bestand und wo nach Behebung der Lageveränderung durch vaginale Fixur Gravidität, aber extrauterin auftrat (Fall 23), hätte bei der Eigenthümlichkeit der topischen Verhältnisse, wie sie hier lagen, vielleicht auch eine Stütze für die Annahme der Wirksamkeit grobmechanischer Verhältnisse geboten, sofern man hier an eine Abknickung des Tubenrohrs denken konnte."

Ich möchte hier kurz auf meine Fälle etwas näher eingehen.

Fall 8: Es handelte sich um eine Frau, die einmal vor 5 Jahren geboren hatte. Nach dem Wochenbett bekam sie Retroversio uteri, starke Beschwerden. 5 Jahre später wurde infolgedessen eine operative Lagecorrectur vorgenommen, Laparotomie, intraperitoneale Verkürzung der Ligg. rotunda. 2 Monate post operationem trat Extrauteringravidität ein. Bei der Laparotomie fand man eine starke Zerrung der schwangeren Tube.

Fall 17: Patientin hat 4 mal geboren, zuletzt vor 10 Jahren. Seither ebenfalls Retroversio uteri. Wegen heftiger Beschwerden wird $91 / 2$ Jahre nach der letzten Entbindung die Ventrofixation vorgenommen. 6 Monate post operationem Eintritt der Tubengravidität. Bei der Laparotomie fand man die sehwangere Tube durch den hochgezogenen Uterus abgeknickt.

In beiden Fällen haben die Frauen, die nach der letzten Entbindung lange Zeit steril geblieben sind, infolge Lagecorrectur des Uterus concipirt; jedoch kam es infolge Abknickung der Tube und dadurch bedingter Verengerung des Tubenlumens zu extrauteriner Einnistung des Eies. Die bei der Operationsautopsie festgestellte Verlagerung und Knickung des Eileiters dürfte mit grosser Wahrscheinlichkeit auf die Lagecorrektur zurückzuführen sein und somit diese Operation direct für die Entstehung der Tubenschwangerschaft verantwortlich gemacht werden können. 


\section{Aeussere Ueberwanderung des Eies.}

Nur in einem einzigen Fall (No. 18) habe ich das Corpus luteum verum im Ovarium der nichtschwangeren Seite gefunden.

\section{Daner der Gravidität.}

Die Extrauteringravidität wurde unterbrochen im

\begin{tabular}{|c|c|c|c|c|c|c|}
\hline 1. & Monat & $13 \mathrm{ma}$ & & Tubarabort & Tubarruptur & intacte $G$ \\
\hline II. & $n$ & 7 & naron & 6 & 1 & \\
\hline III. & , & & $"$ & 2 & 4 & \\
\hline IV. & " & 4 & 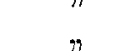 & 0 & 4 & \\
\hline V. & " & $1 n$ & $\Rightarrow$ & 0 & 1 & \\
\hline VII. & $"$ & $1 "$ & $"$ & 0 & 0 & \\
\hline VIII. & n. & 1 & 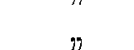 & 0 & 0 & \\
\hline $\mathrm{X}$. & $n$ & $3 n$ & $n$ & 0 & 0 & \\
\hline unklar & & 1 & 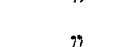 & ? & $?$ & \\
\hline
\end{tabular}

In dem letzten Falle lässt sich nichts Genaues teststellen.

In meinen Fällen war also das Verhältnis von Abort zu Ruptur 17:14 $(=1,2: 1,0)$ d. h. der Abort ist etwas häufiger als die Tubenruptur, und zwar tritt der Abort mebr im I. und II. Schwangerschaftsmonat auf, während im III. und IV. Monat die Tubenruptur, d. h. der äussere Fruehtkapselbruch dominirt.

Diês stimmt überein mit den Resultaten von v. Braun-Fernwald (11), Dobbert (2), Fehling (12), Küstner (4), Lindenthal (13), Martin (3), Runge(1), Orthmann (14), Weindler (15) und Anderen.

\section{Fruchtbefund.}

Es war keine Frucht nachzuweisen. . . in 22 Fällen die Frucht war lebend. . . . . . . " 1 Fall $" \quad "$ frischtodt. . . . . . " 8 Fällen einige Tage todt aber noch ohne Maceration " 3 n macerirt. . . . . . . . . . . . $n 1$ Fall unvollständig verkalkt . . . . . . . $" 1$, 1 skelettirt . . . . . . . . . " 2 Fällen.

Unter den Fällen, die den X. Schwangerschaftsmonat erreichten, liessen sich bei 2 deutlich Deformation an Kopf und unteren Extre- 
mitäten erkennen, wie auch F. v. Winckel (16) in seiner umfangreichen Monographie darauf aufmerksam gemacht hat, dass bei ektopischer Fruchtentwicklung die Föten in der Hälfte aller Fälle Missbildungen aufweisen. Von diesen ist es wieder in $75 \mathrm{pCt}$. der grosse und resistente Kopf, in zweiter Linie (50 pCt.) das Beckenende und die Extremitäten, an denen die Anomalien auftreten. Auch Espenmüller (17) hat hervorgehoben, dass in ,35 pCt. die ektopisch entwickelten Früchte Missbildungen aufweisen. Von den 5 Früchten des VII.-X. Monats präsentirten sich 4 in Beckenendlage. Für diese Lageanomalien und jene Missbildungen glaube ich die Raumbeengung im Fruchtsack als Hauptursache ansprechen zu dürfen.

\section{Symptome.}

a) Menstruationsverhältniss.

Unter meinen Fällen waren 19 Fälle ohne Amenorrhoe, die übrigen 24 ( 1 ist unklar) mit Amenorrhoe verlaufen; es finden sich also auch bei uns zahlreiche Fälle, in denen die Menstruation niemals ausgesetzt hat. So fand auch

\begin{tabular}{|c|c|c|c|c|c|c|c|}
\hline Martin (3) & 25 & Fälle & ohne & und & 32 & mit & Amenorrhoe. \\
\hline Fränkel (17) & 26 & " & $n$ & $n$ & 38 & $n$ & $n$ \\
\hline Küstner (4) & 31 & $n$ & $n$ & 9 & 76 & $n$ & $n$ \\
\hline Dobbert (2) & 21 & 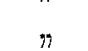 & $n$ & 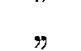 & 29 & $n$ & $n$ \\
\hline Kermauner $(19)$ & 20 & " & . & r & 11 & $n$ & $\eta$ \\
\hline
\end{tabular}

Daraus ergiebt sich, dass ein Ausbleiben der Menstruation keineswegs für die Annahme einer Extrauteringravidität unbedingt erforderlich ist.

\section{b) Blutung.}

Mit Ausnahme von 2 Fällen und einem nicht genau bekanntem Fall wiesen alle sonstigen 35 Fälle atypische Genitalblutung auf. Diese Blutungen setzten nach längerer Amenorrhoe, kurz nach der einmal ausgebliebenen Periode, oder mit der erwarteten Periode ein, oder aber auch schon kurz nach der zuletzt regelmässig dagewesenen Periode. Die Menge des Blutabgangs wechselte, war aber nie sehr profus, jedoch kann bei Vernachlässigung des Falles die langdauernde Blutung eine ziemlich starke Anämie erzeugen. Die Blutung trat bei meinen Fällen immer auf mit Unterbrechung 
der Gravidität; bei Fall 10, der den VII. Schwangerschaftsmont erreichte und bei dem das Kind durch die Operation lebend entwickelt wurde (es blieb 6 Stunden am Leben) war niemals eine Blutung eingetreten. Erst 6 Tage nach der Operation erfolgte eine solche. Die Blutung kann daher anch bei uns als wichtigstes Zeichen für die Unterbrechung einer bestehenden Extrauteringravidität angesehen werden.

c) Unterleibsschmerzen.

Nur in einem Falle (No. 37) blieben diese aus. Sonst waren Leibschmerzen regelmässig vorhanden. Besonders interessant waren nur die Beziehungen zwischen diesen und der Genitalblutung.

Beide kamen gleichzeitig in . . . . 17 Fällen.

Die Blutung ging voraus $n$. . . . . 9 " 9

Der Bauchschmerz ging voraus in . . . 9 "

Unklar . . . . . . . . . . . . 3 "

Fast in der Hälfte der Fälle trat also die Blutung gleichzeitig mit den Leibschmerzen ein. In den Fällen, wo dies nicht der Fall war, betrug indessen die Zeit zwischen dem Auftreten beider Symptome nur einige Tage, höchstens eine Woche.

d) Ohnmacht.

Ohnmachten traten bei uns nur in 5 Fällen ein, und zwar 4 mal bei Tubenruptur $(28,5$ pCt. aller Rupturen), 1 mal bei Tubenabort (6,2 pCt. aller Aborte). Franz (20) hat die Behauptung aufgestellt, dass der Schmerz bei Ruptur stärker sei und plötzlicher auftrete als bei Aborten, dennoch aber der Collaps bei ersteren nicht sehr häufig (27 pCt. der Rupturen) vorkomme, womit meine Berechnung anch übereinstimmt. Andere Autoren, wie Küstner u. s. w., behaupten dagegen, dass anch bei Tubenabort recht häufig Ohnmachten auftreten.

\section{e) Innere Blutung.}

Martin (13) berechnete die Zahl der Fälle mit freier Blutung in die Bauchhöhle auf 40,3 pCt. Unter meinen Fällen fanden sich 14 Fälle, also 36,8 pCt. Die Menge des ergossenen Blutes verhielt sich, wie folgt:

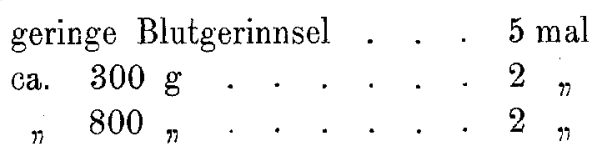




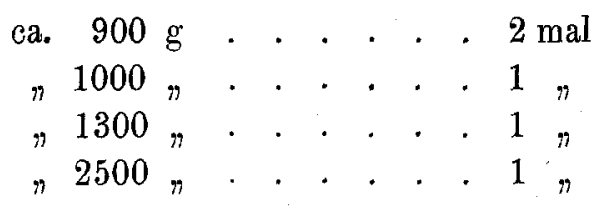

Alle Kranken mit innerer Blutung lassen mehr oder weniger deutlich die Zeichen der Anämie erkennen. Den freien Bluterguss in die Bauchhöhle allein durch die äussere Untersuchung nachzuweisen, ist indessen anch bei grösseren Mengen Blutes sehr schwer, zumal gerade bei reichlichem Erguss durch Reizung des Peritoneums reflectorische Bauchdeckenspannurg und starker Meteorismus sich einstellt.

\section{Therapie.}

Alle unsere Fälle wurden laparotomirt. 8 mal wurde die Mikuliez'sche Tampondrainage angewendet, davon starben 2, bei denen vor der Operation bereits die Fruchtsäcke verjaucht und vereitert waren, an allgemeiner Peritonitis. Einmal wurde per vaginam drainirt; die Patientin genas.

\section{Prognose.}

Die Mortalität bei der Operation der ektopischen Schwangerschaft wird von verschiedenen Autoren verschieden hoch angegeben. Küstner (4) berechnet nur 1,8 pCt., Seidel (21) 14,4 pCt.; Halm und Ledeler (22) 14,8 pCt., Sittner (7 u. 8) 15 pCt., Martin (3) 15,6 pCt., Zuntz (23) 20,3 pCt. und Runge (1) $26,4 \mathrm{pCt}$.

Wir hatten 6 Todesfälle zu verzeichnen (Fall 6, 12, 13, 23, $24,38)=15,7 \mathrm{pCt}$.

In Fall 6 zeigte Patientin schon 1 Woche vor der Operation Reizerscheinungen von Seiten des Peritoneums, Erbrechen, Fieber $\left(38,5^{\circ}\right)$, hohen Puls (110) und starke Empfindlichkeit des Bauches. Sie starb 2 Tage nach der Operation an Peritonitis.

In den Fällen 23 und 24 handelte es sich um Vereiterung und Verjauchung des Fruchtsackes. Auch sie hatten schon vor der Operation hohes Fieber und starke Empfindlichkeit des Abdomens. Sie starben am 3. Tage nach der Operation an Peritonitis.

Fall '12' hatte eine starke innere Blutung, hochgradige 
Anämie. 2 Tage nach der Operation trat Icterus ein. Sie starb am 3. Tage.

Bei Fall 13 betrug die Menge des Blutes in der Bauchhöhle $2500 \mathrm{~g}$. Sie starb kurz nach der Operation.

Fall 28 hatte $900 \mathrm{~g}$ Blut in der Bauchhöhle und litt an Nephritis. Sie starb 1 Tag post operationem.

Wir verloren also 3 Fälle an Peritonitis, 2 an acuter Anämie, 1 an Anämie, complicirt durch Nephritis.

Die wesentlichen Ergebnisse, die sich aus meinen Fällen ableiten lassen, möchte ich zum Schlusse in Folgendem zusammenfassen:

1. Besonders aufällige Unterschiede im Auftreten und im Verlauf der Extrauteringravidität in Japan lassen sich gegenüber deren Verhalten in Europa nicht feststellen.

2. Das Prädilectionsalter ist das 25.--30. Lebensjahr.

3. Frauen, die geboren haben, werden häufiger $(86,8$ pCt.) befallen als Nulliparen.

4. Sehr häufig geht der Extrauteringravidität ein Stadium relativer Sterilität voraus.

5. Relativ häufig ist das Auftreten der ektopisehen Schwangerschaft nach Fehlgeburten.

6. Es ist möglich, dass lagecorrigirende Operationen, wie Ventrofixation oder intraperitoneale Verkürzung der Ligg. rotunda als veranlassendes Moment für die Entstehung der Extrauterinschwangerschaft in Betracht kommen.

7. Die Unterbrechung der Tubargravidität tritt meistens in den ersten Schwangerschaftsmonaten ein, und zwar endet sie im 1. und 2. Monat häufiger durch Abort, im 3. und 4. Nonat häufiger durch Ruptur. Die Zahl der Aborte ist grösser als die der Rupturen.

8. Ektopische Früchte, die das normale Schwangerschaftsende nahezu erreichen, sind zu Lageanomalien und Missbildangen geneigt.

9. Uterusblutung und Bauchschmerz treten meist gleichzeitig auf, oder sie folgen einander in kurzem Zeitabstand (höchstens eine Woche).

10. Ueberwanderung des Eies ist selten (1 mal). 


\section{Literatur.}

1. Runge, Beitrag zur Aetiologie, Symptomatologie und Therapie bei Extrauteringravidität. Dieses Arcbiv. Bd. 70. S. 691.

2. Dobbert, 60 Fälle in früheren kntwicklungsstadien unterbrochener Tubenschwangerschaften. Dieses Archiv. Bd. 66. S. 71.

3. Martin, Die Krankheiten der Eileiter. 1895. Leipzig. S. 303.

4. Küstner, Ueber Extrauterinschwangerschaft. Sammlung klin. Vorträge. No. $244 / 245$.

5. Dührssen, Ueber operative Behandlung; insbesondere die vaginale Köliotomie bei Tubarschwangerschaft, nebst Bemerkungen zur Aetiologie der Tubarschwangerschaft und Beschreibung eines Tubenpolypen. Dieses Archiv. Bd. 54. S. 207.

6. Moebius, Beitrag zur Casuistik der Extrauteringravidität bei lebender und lebensiähiger Frueht. Monatssehr. für Geburtsh. und Gyn. Bd. 15. H. 4. S. 642 .

7. Sittner, Ein Fall von siebenmonatlieher Schwangerschaft ausserhalb der Gebärmutter mit lebendem Kinde. Dieses Archiv. Bd. 64. H. 2. S. 526.

8. Sittner, Weitere Fälle von vorgeschrittener Extrauteringravidität bei lebendem Kinde, behandelt mit Köliotomie. Centralbl. f. Gyn. 1893. No. 2. S. 33.

9. Wormser, Beiträge zur Kenntniss der Extrauteringravidität. Beiträge zur Geburtsh. u. Gyn. Bd. 2. H. 2. S. 298.

10. Werth, Winckel's Handbuch der Geburtshülfe. Bd. II. 2. Theil.

11. v. Braun-Fernwald. Zur Aetiologie, Diagnostik und Therapie der Extrauteringravidität. Dieses Arehiv. Bd. 66. S. 681.

12. Fehling, Die Bedeutung der Tubenruptur und des Tubenabortes für Verlauf, Prognose und Therapie der Tubarschwangerschaft. Zeitschrift für Geburtsh. u. Gyn. Bd. 38. S. 67.

13. Lindenthal, Beitrag zur Therapie der Extrauteringravidität. Monatsschrift f. Geburtsh. u. Gyn. Bd. 11. S. 175.

14. Orthmann, Beitrag zur frühzeitigen Unterbrechung der Tubenschwangerschaft und zur Kenntniss der weiteren Schicksale des Eies. Zeitschrift für Geburtsh. u. Gyn. Bd. 29, S. 61.

15. Weindler, Die Diagnose der frühzeitigen Extrauteringravidität mit Hülfe der Menstruationscurven. Dieses Archiv. Bd. 61. S. 579.

16. v. Winckel, Ueber die Missbildungen von ektopisch entwickelten Früchten und deren Ursachen. Ref. Centralbl. f. Gyn. 1903. No. 13. S. 397.

17. Fränkel, Zur Diagnostik und operativen Behandlung der Tubenschwangerschaft. Dieses Archiv. Bd. 16. S. 197.

18. Espenmüller, Ueber operative Behandlung der Schwangerschaft ausserhalb der Gebärmutter bei lebendem Kinde. Beiträge z. Geburtsh. u. Gyn. Bd. II. H. 2. S. 155 .

19. Kermauner, Beiträge zur Anatomie der Tubenschwangerschaft. Monatsschritt f. Geburtsh. u. Gyn. Bd. XX. Ergänzungsh. S. 634. 
20. Franz, 71. Versammlung deutscher Naturforscher und Aerzte zu München. Ref. Centralbl. f. Gyn. 1899. No. 42. S. 1291.

21. Seidel, Laparotomie bei 90 Fällen von freiem Bluterguss in die Bauchhöhle in Folge unterbrochener Tubenschwangerschaft mit besonderer Berücksichtigung der Prognose. Diss. Berlin. 1903. Cit. Centralbl. f. Gyn. 1905. S. 736 .

22. Halm u. Lederer, Erfahrungen -über rupturirte Extrauterinschwangerschaft mit freiem Bluterguss in die Bauchhöhle. Monatsschrift f. Geburtsh. u. Gyn. Bd. XXV. H. 2.-1.

23. Zuntz, Erfahrungen über Tubargravidität. Dieses Archiv, Bd. 73. H. 1. 